\title{
Estimating Jump Activity Using Multipower Variation
}

DOI:

10.1080/07350015.2020.1784745

\section{Document Version}

Accepted author manuscript

Link to publication record in Manchester Research Explorer

\section{Citation for published version (APA):}

Kolokolov, A. (2020). Estimating Jump Activity Using Multipower Variation. Journal of Business and Economic Statistics. https://doi.org/10.1080/07350015.2020.1784745

\section{Published in:}

Journal of Business and Economic Statistics

\section{Citing this paper}

Please note that where the full-text provided on Manchester Research Explorer is the Author Accepted Manuscript or Proof version this may differ from the final Published version. If citing, it is advised that you check and use the publisher's definitive version.

\section{General rights}

Copyright and moral rights for the publications made accessible in the Research Explorer are retained by the authors and/or other copyright owners and it is a condition of accessing publications that users recognise and abide by the legal requirements associated with these rights.

\section{Takedown policy}

If you believe that this document breaches copyright please refer to the University of Manchester's Takedown Procedures [http://man.ac.uk/04Y6Bo] or contact uml.scholarlycommunications@manchester.ac.uk providing relevant details, so we can investigate your claim.

\section{OPEN ACCESS}




\title{
Estimating Jump Activity Using Multipower Variation
}

\author{
Aleksey Kolokolov* \\ Alliance Manchester Business School, \\ The University of Manchester \\ AlEKSEY.KOLOKOLOV@MANCHESTER.AC.UK
}

July 14,2020

\begin{abstract}
Realized multipower variation, originally introduced to eliminate jumps, can be extremely useful for inference in pure-jump models. This paper shows how to build a simple and precise estimator of the jump activity index of a semimartingale observed at a high frequency by comparing different multipowers. The novel methodology allows to infer whether a discretely observed process contains a continuous martingale component. The empirical part of the paper undertakes a nonparametric analysis of the jump activity of bitcoin and shows that bitcoin is a pure jump process with high jump activity, which is critically different from conventional currencies that include a Brownian motion component.
\end{abstract}

Keywords: bitcoin, jump activity, jumps, high-frequency data, multipower variation

*I thank Roberto Renò, Jean Jacod, Viktor Todorov and Torben Andersen for helpful comments. I am also indebted to Viktor Todorov for making available his Matlab codes for estimation of jump activity index. 


\section{Introduction}

Continuous-time stochastic models based on Itō semimartingales with jumps have been increasingly used in financial econometrics and other areas. The level of vibrancy of an Itō semimartingale is measured by the jump activity (JA) index (Aït-Sahalia and Jacod, 2009). The index takes values in $[0,2]$ and allows the disentangling of different classes of stochastic processes. The JA index is zero for finite activity jump processes (which have a finite number of jumps in any finite interval) and it is equal to two for continuous (local) martingales. Stochastic processes with JA indices in $(0,2)$ are infinitely active pure-jump processes, with paths of infinite variation if the index is larger than unity. In general, a JA index depends on a particular realization of the process on a given interval,

but it is constant with probability one for certain classes of processes. For example, it is 2 for Brownian motion, 0 for a Poisson process and $\beta$ for $\beta$-stable motion. The jump activity of a superposition of different Itō semimartingales is equal to the JA index of the most active component. In particular, if a process is constructed by adding jumps to a continuous process driven by a Brownian motion, its activity index will take a value of 2 independently from the activity of the jumps. It is then clear that estimating the JA index reveals fundamental properties of the process.

Estimating the jump activity index from high-frequency data is of particular interest for financial econometrics, since it makes it possible to distinguish between the two classes of widely used models: Brownian semimartingales plus jumps (with JA index being equal to 2) and pure-jump models (with JA index less than 2). The first class of models is more commonly adopted, see for example, Andersen and Benzoni (2011) and references therein. Pure-jump models have been extensively used for derivative pricing (e.g. Carr and Wu, 2003; Huang and Hung, 2005; Huang and Wu, 2004; Wu, 2006), modeling volatility (e.g. Carr et al., 2003; Todorov and Tauchen, 2011b) and financial prices (e.g. Rydberg, 1997; Barndorff-Nielsen, 1998; Madan et al., 1998; Carr et al., 2002; Jing et al., 2012a). The interest in estimating jump activity indexes of pure-jump processes is not limited to financial applications but it is also relevant, for example, to internet traffic data (Todorov and Tauchen, 2010).

This paper contributes to the existing literature by introducing a new estimator of the 
JA index, which strongly outperforms the existing alternatives (Todorov and Tauchen, 2010, 2011a; Andersen et al., 2015; Todorov, 2015; Aï-Sahalia and Jacod, 2009; Jing et al., 2012b). The new estimator is as easy to use as the two-scale estimator of Todorov and Tauchen (2011a), but it has much smaller finite sample bias and variance, even in comparison with an improved version of the two-scale estimator proposed by Andersen et al. (2015). In practice, this allows estimating jump activity daily, instead of monthly as done in the previous empirical research (Todorov and Tauchen, 2011b), even with moderate sampling frequencies as, for example, a five-minute frequency. The latter is particularly important for estimating the JA index of financial data, as using moderate frequencies allows the mitigation of the adverse impact of market microstructure noise. The new estimator is at least as precise as a very sophisticated estimator of Todorov (2015) (in fact, Monte Carlo experiments in Section 4 show that the new estimator is more precise in many realistic scenarios), but is considerably easier to implement: it requires fewer tuning parameters and much less computational time (roughly 425 times shorter when implemented with the same tuning parameters as in the empirical part of the original paper) for a single estimator. Finally, for pure-jump processes, the new estimator strongly outperforms estimators of Aï-Sahalia and Jacod (2009) and Jing et al. (2012b) in terms of precision, mainly because it is not necessary to set up many tuning parameters (such as thresholds), which heavily affect finite sample performance of the latter estimators.

The idea of the new estimation method consists of comparing realized multipower variations with different powers. The paper shows that, for an appropriate choice of powers, a ratio of different multipower variations converges in probability to a deterministic function of the JA index. Consequently, the index can be estimated by matching this ratio to its theoretical limiting value.

The empirical contribution of this paper consists of analysing jump activity of bitcoin. Previous studies reveal the presence of a non-zero Brownian component in the dynamics of currencies and stock prices (Todorov and Tauchen, 2010; Aï-Sahalia and Jacod, 2010; Cont and Mancini, 2011). This paper provides the evidence that the dynamics of bitcoin is substantially different. Both small and big changes of a bitcoin price appear to be better described by jumps rather than by continuous movements, eliminating the need for 
a continuous martingale component.

In previous literature the inference on Itō semimartingales discretely observed at a high frequency is typically based on realized power variations and their modifications. Realized power variations are defined as the sum of absolute values of high-frequency increments of a process raised to a particular power. Their asymptotic behavior has been extensively studied for continuous Brownian semimartingales with jumps by Barndorff-Nielsen and Shephard (2003, 2004), Jacod (2008), Mancini (2009), Vetter (2010) among others. More recently the results were extended for pure-jump semimartingales by Woerner $(2003,2007)$, Todorov and Tauchen (2010, 2011a), Todorov (2015). In particular, Todorov and Tauchen (2010) proposed an estimator of the JA index based on comparing realized power variations computed over different scales. They show that since the rate at which the realized power variation converges depends solely on the activity of the process, the ratio of the power variations corresponding to different frequencies converges to a deterministic function of the activity index. Their approach is further refined by Todorov and Tauchen (2011a), who derive a Central Limit Theorem for power variations of pure-jump processes, and by Hounyo and Varneskov (2017), who propose bootstrap methods for power variations of locally stable processes. Further, Todorov (2015) introduce a more precise JA estimator based on the real part of the empirical characteristic function of the scaled high-frequency increments of a process, which also utilizes power variation as an auxiliary quantity. A related problem of testing the presence of the Brownian component is considered by AitSahalia and Jacod (2010), Cont and Mancini (2011) and Jing et al. (2012a). However, different authors arrive at opposite conclusions regarding the need to include a continuous component in financial price models. A similar but different problem of estimating JA index of the jumps in the presence of a continuous martingale component was considered by, for example, Aït-Sahalia and Jacod (2009), Jing et al. (2011) and Jing et al. (2012b).

Realized multipower variation was originally introduced by Barndorff-Nielsen and Shephard (2004) to disentangle price volatility due to continuous movements to that due to discontinuous movements. Relevant asymptotic theory was subsequently developed, among others, by Barndorff-Nielsen et al. (2006a,b), Barndorff-Nielsen and Shephard (2006), Corsi et al. (2010) for continuous Brownian semimartingales plus (possibly) a jump process. Re- 
alized multipower variation of pure-jump processes was considered in Woerner (2007) and Hounyo and Varneskov (2017).

The rest of the paper is organized as follows. Section 2 introduces assumptions and notations. Section 3 contains limit theorems for multipower variations, construction of the new estimator, suggestions for practical implementation, and a statistical test for high jump activity. Section 4 is dedicated to the Monte Carlo, while Section 5 reports the empirical application. The proofs are reported in the supplementary Appendix. The supplementary Appendix also contains additional Monte Carlo simulations, explains how to pre-filter real data for annihilating the effect of zeros, and presents two extensions of the developed theory for estimating JA index when (i) JA $\leq 1$; (ii) the observations are contaminated by a microstructure noise.

\section{Setting and assumptions}

Assume that $X$ is a semimartingale defined on a filtered probability space $\left(\Omega, \mathcal{F},(\mathcal{F})_{t \in[0, T]}, \mathcal{P}\right)$ satisfying usual conditions, evolving as

$$
X_{t}=\int_{0}^{t} a_{s} d s+\int_{0}^{t} \sigma_{s-} d L_{s}
$$

where the drift, $a_{t}$, and the stochastic scale, $\sigma_{t}$, are càdlàg processes (specified in the supplementary Appendix), such that for every $t>0,\left|\sigma_{t}\right|,\left|\sigma_{t-}\right|>0$; the driving force $L_{t}$ is a Lévy process specified by either of the two assumptions below.

Assumption 1 (Brownian Semimartingale + Jumps).

$$
L_{t}=W_{t}+J_{t}
$$

where $W_{t}$ is a standard Brownian motion, and $J_{t}$ is an arbitrary pure-jump Lévy process.

Assumption 2 (Pure Jump Semimartingale).

$$
L_{t}=\int_{0}^{t} \int_{\mathbb{R}} \kappa^{\prime}(x) \mu(d s, d x)+\int_{0}^{t} \int_{\mathbb{R}} \kappa(x) \widetilde{\mu}(d x, d s),
$$

where $\kappa(x)$ denotes a continuous truncation function, that is, a continuous function with bounded support such that $\kappa(x)=x$ around the origin, $\kappa^{\prime}(x)=x-\kappa(x)$ and $\mu$ is a Poisson 
random measure on $[0, T] \times \mathbb{R}$ with compensator $\nu(x) d x$, where

$$
\nu(x)=\nu_{1}(x)+\nu_{2}(x)
$$

with

$$
\nu_{1}(x)=\frac{A}{|x|^{\beta+1}}, \quad\left|\nu_{2}(x)\right| \leq \frac{B}{|x|^{\beta^{\prime}+1}}, \quad \text { when }|x| \leq x_{0},
$$

for some $\left.x_{0}>0, B \geq 0, \beta^{\prime}<\beta, \beta \in\right] 1,2[$,

$$
A=\left(\frac{4 \Gamma(2-\beta)|\cos (\beta \pi / 2)|}{\beta(\beta-1)}\right)^{-1} .
$$

Assumptions 1 and 2 include the most widely used models in finance. Under Assumption 1, small high-frequency increments of $X_{t}$ are generated by Brownian motion and are approximately Gaussian, while large variations in $X_{t}$ are due to the jump process, $J_{t}$ (it is assumed that the Brownian component and Lévy jumps share the same "loading" $\sigma_{t}$, which allows shortening the exposition but affects neither the asymptotics nor the inference). The jumps can be arbitrary and include such parametric specifications as, for example, a compound Poisson process, Gamma process or locally stable processes from Assumption 2. Assumption 2 is similar to the one used by Todorov and Tauchen (2010, 2011a) and in related papers. It implies that the pure-jump process is locally stable, that is, its small time increments are similar to those of $\beta$-stable process. The assumption is satisfied by many pure-jump processes used in finance, for example, the tempered stable process. Note that the value of $\beta$ in Assumption 2 is assumed to be larger than 1 . Hence, $X_{t}$ is an infinite variation jump process. Inference for finite variation pure-jump processes is considered in the supplementary Appendix. Equation (6) means that the Lévy measure $\nu_{1}$ in Assumption 2 is the Lévy measure of the standard $\beta$-stable process. The choice of $A$ can be made without loss of generality as the leading (at a high frequency) component of the observed process is an integral of $\sigma_{t}$ with respect to $L_{t}$, but the two processes $\left(\sigma_{t}\right.$ and $\left.L_{t}\right)$ are never observed separately. Under both Assumptions 1 and 2 the stochastic scale $\sigma_{t}$ is specified as a semimartingale with jumps. The precise assumption is completely borrowed from Todorov and Tauchen (2011a) and it is stated in the supplementary Appendix.

The jump activity of $X$ is measured by the jump activity index defined as the smallest 
power for which the realized power variation does not explode:

$$
\mathrm{JA}=\inf \left\{r>0: \operatorname{plim}_{\Delta_{n} \rightarrow 0} \sum_{s=1}^{\left[t / \Delta_{n}\right]}\left|X_{s \Delta_{n}}-X_{(s-1) \Delta_{n}}\right|^{r}<\infty\right\} .
$$

The index takes its values in $[0,2]$. In general, JA depends on a particular realization of the process, but for a process satisfying Assumption 2, JA $=\beta$ for all paths; for a process satisfying Assumption 1, JA = 2 for all paths.

Estimation of the JA index is carried out by observing a discretized path of $X$ on a finite time interval. From now on, assume that $n+1$ equally spaced observations of $X$ are recorded in the interval $[0, t]$ (with fixed $t$ ). Set $\Delta_{n}=\lfloor t / n\rfloor$ and define the evenly sampled high-frequency increments as

$$
\Delta_{j} X=X_{j \Delta_{n}}-X_{(j-1) \Delta_{n}}, \quad j=1, \ldots, n
$$

\section{Jump activity estimation}

\subsection{Construction of the estimator}

Let $r=\left[r_{1}, \ldots, r_{m}\right]$ be a vector of real positive numbers and define realized multipower variation $\mathrm{MV}(r)$ as

$$
\operatorname{MV}(r)=\sum_{i=1}^{n-m+1}\left|\Delta_{i} X\right|^{r_{1}} \ldots\left|\Delta_{i+m-1} X\right|^{r_{m}}
$$

If powers $r_{i}$ are sufficiently small, realized multipower variation admits a Law of Large Numbers independently of whether $X$ is a pure jump or a Brownian semimartingale.

\section{Theorem 1.}

(I) Let $X$ be a pure-jump process satisfying Assumption 2. Assume that $r_{i}<\beta, i=$ $1, \ldots, m$. As $n \rightarrow \infty$

$$
n^{r_{+} / \beta-1} \operatorname{MV}(r) \stackrel{p}{\longrightarrow} \prod_{j=1}^{m} \mu_{r_{j}}(\beta) \int_{0}^{t}\left|\sigma_{s}\right|^{r_{+}} d s,
$$

where $\mu_{r}(\beta)=\mathbf{E}\left[|S|^{r}\right]$ with $S$ being a standard symmetric $\beta$-stable random variable, $r_{+}=r_{1}+\ldots+r_{m}$. 
(II) Let $X$ be a process satisfying Assumption 1. Assume that $r_{i}<2, i=1, \ldots, m$. As $n \rightarrow \infty$,

$$
n^{r+/ 2-1} \mathrm{MV}(r) \stackrel{p}{\longrightarrow} \prod_{j=1}^{m} \mu_{r_{j}} \int_{0}^{t}\left|\sigma_{s}\right|^{r_{+}} d s
$$

where $\mu_{r}=\mathbf{E}\left[|U|^{r}\right]$ with $U$ being a standard normal random variable, $r_{+}=r_{1}+\ldots+r_{m}$.

Remark 1. Part (II) of the Theorem 1 is a well known result for multipower variations (Barndorff-Nielsen et al., 2006a; Jacod and Protter, 2012). If a process X satisfies Assumption 1 and has continuous sample paths, it holds for any positive powers (Barndorff-Nielsen et al., 2006b). Special cases of part (I) were previously established by Woerner (2007) and Hounyo and Varneskov (2017).

The fractional absolute moments of a symmetric standard $\beta$-stable random variable for $-1<r<\beta$ (Shanbhag and Sreehari, 1977) do not converge to the corresponding moments of a standard normal random variable as $\beta \rightarrow 2$ :

$$
\mu_{r}(\beta)=\frac{2^{r} \Gamma((1+r) / 2) \Gamma(1-r / \beta)}{\Gamma(1-r / 2) \Gamma(1 / 2)}, \quad \text { and } \quad \mu_{r}=\frac{2^{r / 2} \Gamma((1+r) / 2)}{\Gamma(1 / 2)} .
$$

Despite of the fact that $\mu_{r}(2) \neq \mu_{r}$,

$$
\prod_{j=1}^{m} \frac{\mu_{r_{j}^{(1)}}(2)}{\mu_{r_{j}^{(2)}}(2)}=\prod_{j=1}^{m} \frac{\mu_{r_{j}^{(1)}}}{\mu_{r_{j}^{(2)}}}
$$

provided that $r_{+}^{(1)}=r_{+}^{(2)}$, because $\mu_{r}(2)=\frac{2^{r} \Gamma((1+r) / 2)}{\Gamma(1 / 2)}=2^{r / 2} \mu_{r}$, and the constant factor $2^{r}$ cancels when dividing. Consequently, by Theorem 1 , if $r^{(1)}$ and $r^{(2)}$ denote two different sets of powers with $r_{+}^{(1)}=r_{+}^{(2)}$,

$$
\frac{\operatorname{MV}\left(r^{(1)}\right)}{\operatorname{MV}\left(r^{(2)}\right)} \stackrel{p}{\longrightarrow} \prod_{j=1}^{m} \frac{\mu_{r_{j}^{(1)}}(\beta)}{\mu_{r_{j}^{(2)}}(\beta)}
$$

where $\mu_{r}(\beta)$ denotes a formal function of $\beta$ defined by expression (12). Consequently, a consistent (for both pure-jump and Brownian semimartingales with jumps) estimator of $\beta$ can be defined as a root of the function

$$
Q\left(\beta ; X, r^{(1)}, r^{(2)}\right)=\frac{\operatorname{MV}\left(r^{(1)}\right)}{\operatorname{MV}\left(r^{(2)}\right)}-\prod_{j=1}^{m} \frac{\mu_{r_{j}^{(1)}}(\beta)}{\mu_{r_{j}^{(2)}}(\beta)},
$$

provided that a unique root exists. 
A simple example of a consistent estimator is based on bipower variations $(m=2)$ with powers:

$$
r^{(1)}=\left[r_{+} 0\right], \quad r^{(2)}=\left[r_{+} / 2 \quad r_{+} / 2\right],
$$

for some $r_{+}<\beta$. For this choice of powers, one can deduce from the first equation in (12) by straightforward algebra that the function $Q\left(\beta ; X,\left[r_{+}, 0\right],\left[r_{+} / 2, r_{+} / 2\right]\right)$ can be expressed as

$$
Q\left(\beta ; X, r_{+}\right)=\frac{\mathrm{MV}\left(\left[r_{+}, 0\right]\right)}{\operatorname{MV}\left(\left[r_{+} / 2, r_{+} / 2\right]\right)}-\gamma\left(\beta ; r_{+}\right)
$$

where

$$
\gamma\left(\beta ; r_{+}\right)=\widetilde{\gamma}\left(r_{+}\right) \cdot \frac{\Gamma\left(1-\frac{r_{+}}{\beta}\right)}{\Gamma^{2}\left(1-\frac{r_{+}}{2 \beta}\right)}, \quad \text { and } \quad \widetilde{\gamma}\left(r_{+}\right)=\frac{\Gamma\left(\frac{1}{2}\right) \Gamma\left(\frac{1}{2}+\frac{r_{+}}{2}\right) \Gamma^{2}\left(1-\frac{r_{+}}{4}\right)}{\Gamma\left(1-\frac{r_{+}}{2}\right) \Gamma^{2}\left(\frac{1}{2}-\frac{r_{+}}{4}\right)} .
$$

Due to the properties of the Gamma function, $\gamma\left(\beta ; r_{+}\right)$is a decreasing function of $\beta$ on $\left(r_{+},+\infty\right)$ satisfying:

$$
\lim _{\beta \rightarrow r_{+}+} \gamma\left(\beta ; r_{+}\right)=+\infty, \quad \lim _{\beta \rightarrow+\infty} \gamma\left(\beta ; r_{+}\right)=\widetilde{\gamma}\left(r_{+}\right)
$$

Consequently, there exists the unique zero of $Q\left(\beta ; X, r_{+}\right)$on the interval $\left(r_{+},+\infty\right)$, provided that the following inequality holds:

$$
\frac{\operatorname{MV}\left(\left[r_{+}, 0\right]\right)}{\operatorname{MV}\left(\left[r_{+} / 2, r_{+} / 2\right]\right)}>\widetilde{\gamma}\left(r_{+}\right)
$$

The above inequality constitutes the condition of the existence of the JA estimator defined as the root of $Q\left(\beta ; X, r_{+}\right)$on $\left(r_{+},+\infty\right)$. Due to the convergence (14), such an estimator always exists asymptotically and it is larger than $r_{+}$by construction.

The conditions for the existence of a unique zero of $Q\left(\beta ; X, r^{(1)}, r^{(2)}\right)$ are not straightforward for general powers. Moreover, using bipower variation $(m=2)$ instead of a multipower variation with many powers is beneficial for minimizing the finite sample bias due to the variation of the stochastic scale. Therefore, in the rest of the paper JA estimation is based on the powers of the form (16). That is, the JA estimator is defined as follows.

Definition 1. A JA estimator $\widehat{\beta}=\widehat{\beta}\left(r_{+}\right)$is the (pointwise in $\omega$ ) solution of the equation

$$
\frac{\operatorname{MV}\left(\left[r_{+}, 0\right]\right)}{\operatorname{MV}\left(\left[r_{+} / 2, r_{+} / 2\right]\right)}-\frac{\mu_{r_{+}}(\beta)}{\mu_{r_{+} / 2}(\beta) \mu_{r_{+} / 2}(\beta)}=0
$$

provided that the unique solution on $\left(r_{+},+\infty\right)$ exists. 
Corollary 1. If either $X_{t}$ is a pure-jump process satisfying Assumption 2 with $1<\beta<2$ and $r_{+}<\beta$, or $X_{t}$ satisfy Assumption 1 and $r_{+}<2$, as $n \rightarrow \infty$,

$$
\widehat{\beta}\left(r_{+}\right) \stackrel{p}{\longrightarrow} J A
$$

where JA is the jump activity index of $X_{t}$ defined by (2).

Remark 2. One can slightly modify the definition of the estimator in order to guarantee its existence in finite samples. For example, a (modified) estimator can be defined as the solution of the equation

$$
\widetilde{Q}\left(\beta ; X, r_{+}\right)=0
$$

where $\widetilde{Q}\left(\beta ; X, r_{+}\right)$is a smooth function of $\beta$, such that, for some $\beta^{\dagger}>2$,

$$
\begin{cases}\widetilde{Q}\left(\beta ; X, r_{+}\right)=Q\left(\beta ; X, r_{+}\right), & \text {for } \beta<\beta^{\dagger}, \\ \widetilde{Q}\left(\beta ; X, r_{+}\right) \rightarrow-\infty, & \text { as } \beta \rightarrow+\infty .\end{cases}
$$

The asymptotic properties of the modified estimator will be similar to the limiting properties

of $\widehat{\beta}$, but the asymptotic variance, which would depend on the choice of $\beta^{\dagger}$ and on how $\widetilde{Q}\left(\beta ; X, r_{+}\right)$is defined for $\beta>\beta^{\dagger}$. The modification, however, is not employed in the rest of the paper, as it appears that $\widehat{\beta}$ exists in all Monte Carlo simulations presented below as well as in the empirical application.

\subsection{Central Limit Theorem}

This section presents the joint Central Limit Theorem for realized multipower variations with different powers. Then, the CLT is used to derive the asymptotic distribution of the JA estimator.

Theorem 2. Let $r^{(1)}=\left[r_{1}^{(1)}, \ldots, r_{m}^{(1)}\right]$ and $r^{(2)}=\left[r_{1}^{(2)}, \ldots, r_{m}^{(2)}\right]$ be two sets of powers. Assume that either of the following assumptions holds:

(I) $X$ is a pure-jump process satisfying Assumption 2 with $\beta>\sqrt{2}$ and $\beta^{\prime}<\beta / 2$,

$$
\frac{2-\beta}{2(\beta-1)} \vee \frac{\beta-1}{2} \vee \frac{\beta \beta^{\prime}}{2\left(\beta-\beta^{\prime}\right)}<r_{j}^{(i)}<\frac{\beta}{2}, \quad i=1,2, j=1, \ldots, m .
$$


(II) $X$ is a process satisfying Assumption 1 with the jump part, J, being a finite variation pure-jump process with JA index $\beta_{J}<1$,

$$
\frac{\beta_{J}}{2-\beta_{J}}<r_{j}^{(i)}<1, \quad i=1,2, j=1, \ldots, m
$$

Then, as $n \rightarrow \infty$,

$$
\sqrt{n}\left(\begin{array}{c}
n^{\frac{r_{+}^{(1)}}{\beta}-1} \mathrm{MV}\left(r^{(1)}\right)-\prod_{j=1}^{m} \mu_{r_{j}^{(1)}}(\beta) \int_{0}^{t}\left|\sigma_{s}\right|^{r_{+}^{(1)}} d s \\
n^{\frac{r_{+}^{(2)}}{\beta}-1} \mathrm{MV}\left(r^{(2)}\right)-\prod_{j=1}^{m} \mu_{r_{j}^{(2)}}(\beta) \int_{0}^{t}\left|\sigma_{s}\right|^{r_{+}^{(2)}} d s
\end{array}\right) \stackrel{L-s t}{\longrightarrow} \Sigma^{1 / 2} \cdot \mathbf{Z}
$$

where $\stackrel{\text { L-st }}{\longrightarrow}$ denotes stable convergence in law, $\mathbf{Z}$ is a two-dimensional standard normal random variable defined on an extension of the original probability space, and the covariance matrix $\Sigma$ is defined as:

$$
\Sigma_{i j}=\Psi_{i j}(\beta) \int_{0}^{t}\left|\sigma_{s}\right|^{r_{+}^{(i)}+r_{+}^{(j)}} d s
$$

with

$$
\begin{aligned}
\Psi_{i j}(\beta) & =\prod_{k=1}^{m} \mu_{r_{k}^{(i)}+r_{k}^{(j)}}(\beta)+\sum_{k=1}^{m-1} \prod_{\ell=1}^{k} \mu_{r_{\ell}^{(i)}}(\beta) \prod_{\ell=1}^{m-k} \mu_{r_{k+\ell}^{(i)}+r_{\ell}^{(j)}}(\beta) \prod_{\ell=m-k+1}^{m} \mu_{r_{\ell}^{(j)}}(\beta) \\
& +\sum_{k=1}^{m-1} \prod_{\ell=1}^{k} \mu_{r_{\ell}^{(j)}}(\beta) \prod_{\ell=1}^{m-k} \mu_{r_{k+\ell}^{(j)}+r_{\ell}^{(i)}}(\beta) \prod_{\ell=m-k+1}^{m} \mu_{r_{\ell}^{(i)}}(\beta)-(2 m-1) \prod_{k=1}^{m} \mu_{r_{k}^{(i)}}(\beta) \mu_{r_{k}^{(j)}}(\beta),
\end{aligned}
$$

using the convention $\mu_{r}(2)=\mu_{r}$.

Remark 3. Theorem 2 continues to hold if the sets of powers $r^{(i)}=\left[r_{1}^{(i)}, \ldots, r_{m}^{(i)}\right]$ are nonnegative with at least one positive power for both $i=1,2$. In that case, formula (27) holds as $\mu_{0}(\beta)=1, \forall \beta \in(0,2]$.

Remark 4. Theorem 2 (I) imposes several restrictions on the powers $r_{j}^{(i)}$ and $\beta$. The restriction $r_{j}^{(i)}>\frac{\beta \beta^{\prime}}{2\left(\beta-\beta^{\prime}\right)}$ is required for eliminating the contribution of the less active residual jump component and would be relaxed if $L_{t}$ is $\beta$-stable motion. The restrictions $r_{j}^{(i)}>\frac{2-\beta}{2(\beta-1)}$, $r_{j}^{(i)}>\frac{\beta-1}{2}$ and $\beta>\sqrt{2}$ eliminate the contribution of the drift. $r_{j}^{(i)}>\frac{\beta-1}{2}$ is relaxed when $X_{t}$ is a Lévy process.

Theorem 2 implies that the JA estimator in Definition 1 is asymptotically mixed normal in both pure-jumps and Brownian cases provided that the powers are sufficiently small. More precisely, the following corollary holds. 
Corollary 2. Let $\widehat{\beta}\left(r_{+}\right)$denote the JA estimator introduced in Definition 1 . Under the assumptions of the Theorem 2, as $n \rightarrow \infty$,

$$
\sqrt{n}\left(\widehat{\beta}\left(r_{+}\right)-\beta\right) \stackrel{L-s t}{\longrightarrow} \sqrt{\Sigma(\beta)} \cdot \mathbf{U}
$$

where $\mathbf{U}$ is standard normal random variable,

$$
\Sigma(\beta)=\widetilde{\vartheta}(\beta) \cdot \frac{\int_{0}^{t}\left|\sigma_{s}\right|^{2 r_{+}} d s}{\left(\int_{0}^{t}\left|\sigma_{s}\right|^{r_{+}} d s\right)^{2}},
$$

and

$$
\begin{aligned}
& \tilde{\vartheta}(\beta)=\frac{2 \mu_{r_{+} / 2}^{2}(\beta) \mu_{r_{+}}^{3}(\beta)+\mu_{r_{+}}^{4}(\beta)+\mu_{r_{+} / 2}^{4}(\beta) \mu_{2 r_{+}}(\beta)-4 \mu_{r_{+} / 2}^{3}(\beta) \mu_{r_{+}}(\beta) \mu_{3 r_{+} / 2}(\beta)}{\mu_{r_{+} / 2}^{2}(\beta)\left(\mu_{r_{+} / 2}(\beta) \mu_{r_{+} / 2}^{\prime}(\beta)-2 \mu_{r_{+}}(\beta) \mu_{r_{+}}^{\prime}(\beta)\right)^{2}}, \\
& \widetilde{\vartheta}(\beta)=\frac{2 \mu_{r_{+} / 2}^{2}(\beta) \mu_{r_{+}}^{3}(\beta)+\mu_{r_{+}}^{4}(\beta)-4 \mu_{r_{+} / 2}^{3}(\beta) \mu_{r_{+}}(\beta) \mu_{3 r_{+} / 2}(\beta)+\mu_{r_{+} / 2}^{4}(\beta) \mu_{2 r_{+}}(\beta)}{\mu_{r_{+} / 2}^{2}(\beta)\left(\mu_{r_{+} / 2}(\beta) \mu_{r_{+} / 2}^{\prime}(\beta)-2 \mu_{r_{+}}(\beta) \mu_{r_{+}}^{\prime}(\beta)\right)^{2}},
\end{aligned}
$$

where

$$
\mu_{r}^{\prime}(\beta)=\frac{2^{r} r \Gamma^{\prime}(1-r / \beta) \Gamma(r / 2+1 / 2)}{\beta^{2} \Gamma(1-r / 2) \Gamma(1 / 2)},
$$

and $\Gamma^{\prime}$ denotes the derivative of the Gamma function.

Remark 5. The terms in the asymptotic variance of the JA estimator can be consistently estimated by multipower variations, as follows from Theorem 1.

Corollary 2 implies that asymptotic variance of the new JA estimator has similar structure to the variances of the two-scale jump activity estimators proposed by Todorov and Tauchen (2010) and Andersen et al. (2015) (defined in equation (40) below). The asymptotic variance of the latter estimator (based on the realized power variation with the power $\left.r_{+}\right)$takes the form

$$
\left(\frac{\beta^{2}}{\mu_{r_{+}}(\beta) r_{+} \log (2)}\right)^{2} \widetilde{\Xi} \cdot \frac{\int_{0}^{t}\left|\sigma_{s}\right|^{2 r_{+}} d s}{\left(\int_{0}^{t}\left|\sigma_{s}\right|^{r_{+}} d s\right)^{2}},
$$

where $\widetilde{\Xi}=\Xi^{(1,1)}-2^{1-r_{+} / \beta} \Xi^{(1,2)}+2^{-2 r_{+} / \beta} \Xi^{(2,2)}, \Xi=\Sigma_{0}\left(r_{+}, \beta\right)+\Sigma_{1}\left(r_{+}, \beta\right)+\Sigma_{1}^{\prime}\left(r_{+}, \beta\right)$ and $\Sigma_{j}\left(r_{+}, \beta\right)=\mathbf{E}\left[\mathbf{Z}_{1} \mathbf{Z}_{1+j}^{\prime}\right], j=0,1$, with $\mathbf{Z}_{i}=\left(\left|S_{i}\right|^{r_{+}}-\mu_{r_{+}}(\beta),\left|S_{i}+S_{i+1}\right|^{r_{+}}-2^{r_{+} / \beta} \mu_{r_{+}}(\beta)\right)^{\prime}$, and $S_{0}, S_{1}, \ldots$ are i.i.d. $\beta$-stable random variables. That is, the difference in the asymptotic standard deviations between the new JA estimator and the two-scale estimator (if the same 
tuning parameter, $r_{+}$, is used for both) is driven by the constant factors multiplying the

ratio $\frac{\int_{0}^{t}\left|\sigma_{s}\right|^{2 r}+d s}{\left(\int_{0}^{t}\left|\sigma_{s}\right|^{r}+d s\right)^{2}}$, which depend on $\beta$ and $r_{+}$, but not on the stochastic scale. Figure 1 compares the square roots of the constant factors (which are, in fact, equal to the asymptotic standard deviations of the estimators when $X_{t}$ is a Lévy process). The figure shows that the asymptotic standard deviation of the new mutipower-based estimator is typically smaller than that of the two-scale estimator. The difference between the two is particularly large in the limiting case, $\beta=2$, which corresponds to the situation when $X_{t}$ includes the Brownian component.

Remark 6. The asymptotic standard deviation of the two-scale estimator depends on the expectations $\mathbf{E}\left[\left|S_{1}\right|^{r_{+}}\left|S_{1}+S_{2}\right|^{r_{+}}\right]$and $\mathbf{E}\left[\left|S_{1}+S_{2}\right|^{r_{+}}\left|S_{2}+S_{3}\right|^{r_{+}}\right]$, where $S_{1}, S_{2}$ and $S_{3}$ are stable random variables, which are not known in closed form to the best of my knowledge. Therefore, they are computed using the Monte Carlo method. The computation of asymptotic standard deviation of the new estimator does not require Monte Carlo.

\subsection{Adaptive implementation in practice}

How to choose $r_{+}$in practice? In the Lévy case, it is natural to choose $r_{+}$by minimizing the asymptotic standard deviation $\widetilde{\vartheta}(\beta)$, which depends on an unknown JA index. Hence, the estimator can be applied in two steps. First, a preliminary estimate of JA, say $\widehat{\beta}_{1}$, corresponding to a small value of $r_{+}<1$ (which guarantees the consistency), is computed. Next, a second-step estimator is implemented with $r_{+}$minimizing $\widetilde{\vartheta}\left(\widehat{\beta}_{1}\right)$. However, in short samples this strategy can be improved by minimizing the (simulated) sample variance of the estimator for a $\beta$-stable process sampled at a fixed frequency. Table 1 reports the optimal values of $r_{+}$for sampling frequencies ranging from $n=39$ to $n=23400$ (roughly corresponding to 10-minute and one-second sampling of a stock price in a 6.5 hours trading day, respectively) and different JA indexes. The last row of the table provides the asymptotically optimal $r_{+}$minimizing $\widetilde{\vartheta}(\beta)$. It follows that for all considered JA indexes, the larger values of $r_{+}$deliver more precise estimators when $n$ is small. As $n$ increases, the optimal $r_{+}$converges to the value minmizing the limiting standard deviation. The convergence is, however, quite slow, especially for larger JA indexes. Hence, in the $\beta$-stable 

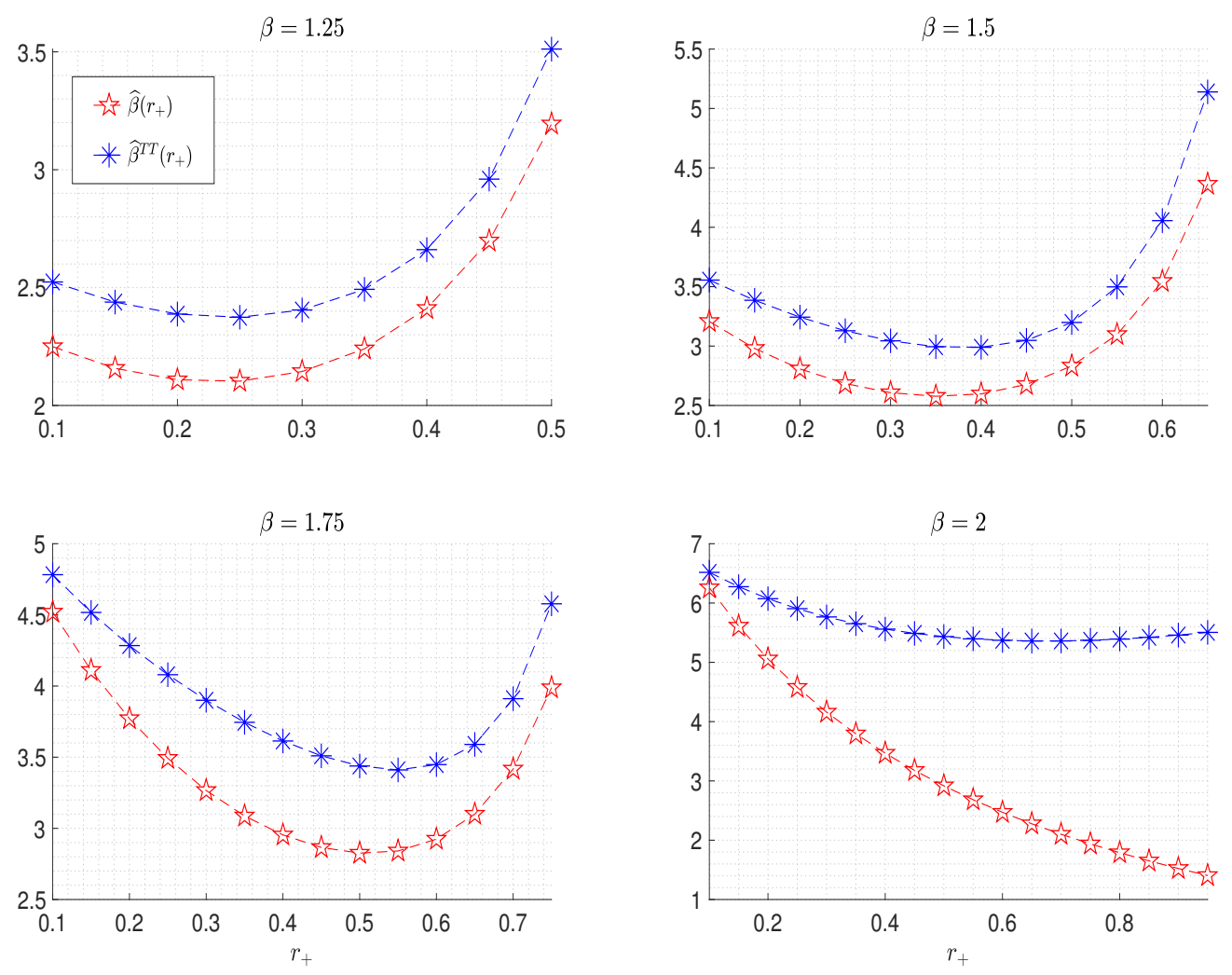

Figure 1: Asymptotic standard deviations of $\widehat{\beta}\left(r_{+}\right)$and the two-scale estimator of Andersen et al. (2015) (denoted $\widehat{\beta}^{T T}\left(r_{+}\right)$), as a function of $r_{+}$when $X_{t}$ is a Lévy process. The limiting case $\beta=2$ corresponds to the case when $L_{t}$ is equal to the standard Brownian motion. 
Table 1: Optimal $r_{+}$for $\beta$-stable Lévy processes.

\begin{tabular}{|c|c|c|c|c|c|c|c|c|c|c|}
\hline & \multicolumn{10}{|c|}{ Jump Activity index, $\beta$} \\
\hline$n$ & 1.05 & 1.15 & 1.25 & 1.35 & 1.45 & 1.55 & 1.65 & 1.75 & 1.85 & 1.95 \\
\hline 39 & 0.525 & 0.575 & 0.625 & 0.675 & 0.725 & 0.775 & 0.825 & 0.875 & 0.925 & 0.975 \\
\hline 78 & 0.525 & 0.575 & 0.625 & 0.675 & 0.725 & 0.775 & 0.825 & 0.875 & 0.925 & 0.975 \\
\hline 144 & 0.350 & 0.450 & 0.625 & 0.675 & 0.725 & 0.775 & 0.825 & 0.875 & 0.925 & 0.975 \\
\hline 288 & 0.250 & 0.300 & 0.350 & 0.450 & 0.550 & 0.775 & 0.825 & 0.875 & 0.925 & 0.975 \\
\hline 390 & 0.200 & 0.250 & 0.350 & 0.400 & 0.500 & 0.600 & 0.825 & 0.875 & 0.925 & 0.975 \\
\hline 780 & 0.200 & 0.250 & 0.300 & 0.350 & 0.400 & 0.450 & 0.600 & 0.700 & 0.925 & 0.975 \\
\hline 1440 & 0.143 & 0.200 & 0.300 & 0.300 & 0.350 & 0.400 & 0.500 & 0.600 & 0.700 & 0.975 \\
\hline 2340 & 0.143 & 0.200 & 0.232 & 0.300 & 0.350 & 0.400 & 0.500 & 0.600 & 0.700 & 0.975 \\
\hline 2880 & 0.143 & 0.200 & 0.250 & 0.300 & 0.350 & 0.400 & 0.500 & 0.550 & 0.700 & 0.975 \\
\hline 8640 & 0.143 & 0.187 & 0.232 & 0.300 & 0.350 & 0.400 & 0.450 & 0.550 & 0.600 & 0.850 \\
\hline 23400 & 0.143 & 0.187 & 0.250 & 0.279 & 0.329 & 0.400 & 0.450 & 0.550 & 0.650 & 0.800 \\
\hline$\infty$ & 0.143 & 0.187 & 0.232 & 0.279 & 0.329 & 0.384 & 0.444 & 0.513 & 0.599 & 0.735 \\
\hline
\end{tabular}

Note. The table reports the values of $r_{+}$minimizing sample standard deviation of the JA estimator $\widehat{\beta}\left(r_{+}\right)$when $X_{t}$ is Lévy $\beta$-stable process for different sample frequencies (rows) and different JA indexes (columns). The minimum is found by a grid search over the values $r_{+}$ranging from 0.005 to $\beta / 2$ with step 0.005 and the theoretically optimal value. The last row reports the values of $r_{+}$minmizing the theoretical asymptotic standard deviation.

Lévy case, the second-step estimator ought to be implemented with $r_{+}$provided by Table 1 for a given frequency and the JA index estimated at the first step.

Choosing $r_{+}$in a general case is more challenging as the standard deviation of the estimator depends on a particular realization of the stochastic scale $\sigma$. In general, no $r_{+}$, which would deliver the smallest variance for every realization of $\sigma$, exists. Moreover, Theorem 2 requires $r_{+}$to be not very small: it should satisfy $\frac{\beta \beta^{\prime}}{2\left(\beta-\beta^{\prime}\right)}<r_{+}$in the pure-jump case or $\frac{\beta_{J}}{2-\beta_{J}}<r_{+}$under the presence of the Brownian component, where $\beta^{\prime}$ and $\beta_{J}$ are the JA indexes of respectively the residual jump component and the pure-jump part. In practice, neither $\beta^{\prime}$ nor $\beta_{J}$ are known and they are hard to estimate.

Consequently, for implementing the estimator in practice the following conservative rule of thumb is recommended. First, a preliminary estimator $\beta\left(r_{+}\right)$is computed using a small 
value of $r_{+}$. Next, the second step-estimator is computed as $\widehat{\beta}\left(\widehat{\beta}\left(r_{+} / 2 \wedge 1\right)\right)$. This strategy is beneficial for short sample performance of the estimator in a Lévy case (as follows from Table 1) and insures the conditions $\frac{\beta \beta^{\prime}}{2\left(\beta-\beta^{\prime}\right)}<r_{+}$and $\frac{\beta_{J}}{2-\beta_{J}}<r_{+}$of the Central Limit Theorem 2 in general case. In what follows the two-step estimator is denoted simply by $\widehat{\beta}$.

\subsection{A formal test for high jump activity}

The estimation theory developed above allows the construction of one-sided statistical tests for given levels of JA index. Consider testing the following hypothesis:

$$
\mathcal{H}_{0}: \beta \leq \beta^{\diamond} \quad \text { v.s. } \quad \mathcal{H}_{1}: \beta>\beta^{\diamond}
$$

where $\beta^{\diamond}$ is a given number from the interval $(1,2)$. The test statistic can be deduced from Corollary 2 by replacing the unknown asymptotic variance of $\widehat{\beta}\left(r_{+}\right)$with an estimator based on multipower variation. For some $r_{+}<\frac{\beta^{\diamond}}{2}$, define the test statistics as

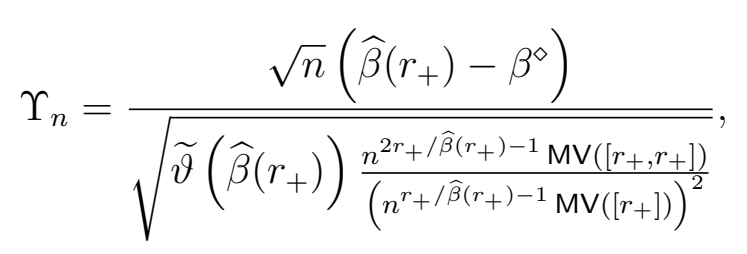

where $\widetilde{\vartheta}(\beta)$ is the function defined by equation $(30)$.

Corollary 3. Under the assumptions of Theorem 2, as $n \rightarrow \infty$,

$$
\begin{cases}\Upsilon_{n} \stackrel{L-s t}{\longrightarrow} \mathcal{N}(0,1), & \text { if } \beta=\beta^{\diamond}, \\ \Upsilon_{n} \stackrel{p}{\longrightarrow}+\infty, & \text { if } \beta>\beta^{\diamond}, \\ \Upsilon_{n} \stackrel{p}{\longrightarrow}-\infty, & \text { if } \beta<\beta^{\diamond},\end{cases}
$$

where $\mathcal{N}(0,1)$ denotes standard normal distribution.

The above corollary implies that if $\Upsilon_{n}$ falls into the critical region $\left(\phi_{1-\alpha},+\infty\right)$, where $\phi_{1-\alpha}$ denotes the $(1-\alpha)$-quantile of standard normal distribution, $\mathcal{H}_{0}$ ought to be rejected in favor of $\mathcal{H}_{1}$, and $\lim _{n \rightarrow \infty} \mathcal{P}\left\{\Upsilon_{n} \in\left(\phi_{1-\alpha},+\infty\right) \mid \mathcal{H}_{0}\right\} \leq \alpha$. The test can be helpful for distinguishing pure-jump processes with high JA index from those containing a Brownian component: in the presence of a Brownian motion $\mathcal{H}_{0}$ ought to be rejected for any $\beta^{\diamond}<2$. The power of the test in finite samples is investigated in Section 4 . 


\section{Monte Carlo}

In order to test finite sample performance of the new estimator, I simulate sample paths of a random process specified as:

$$
d X_{t}=a d t+\sigma_{t} d L_{t}
$$

where $L_{t}$ either includes a Brownian motion or equals to a pure-jump process. In finite samples, the properties of jump activity estimators may depend on the data-generating process as well as on the sampling frequency. Therefore different specifications of $X_{t}$ and different sampling frequencies are considered.

First, $X_{t}$ is specified as in Todorov (2015). $X_{t}$ is defined by equation (36) with $a=0$ and the stochastic scale, $\sigma_{t}$, evolving as:

$$
d \sigma_{t}=-0.03 \sigma_{t} d t+d Z_{t},
$$

where $L_{t}$ and $Z_{t}$ are two independent Lévy processes with Lévy densities given by $\nu_{L}(x)=$ $e^{-\lambda|x|}\left(\frac{A_{0}}{|x|^{1+\beta}}+\frac{A_{1}}{|x|^{1+\beta / 3}}\right)$ and $\nu_{Z}(x)=0.0293 \frac{e^{-3 x}}{x^{1.5}} \mathbf{1}_{x>0}$ respectively. That is, $X_{t}$ is a mixture of tempered stable processes with a stochastic scale following a Lévy-driven OrnsteinUhlenbeck process. The JA index of $X_{t}$ coincides with the parameter $\beta$. Four different levels of jump activity, corresponding to $\beta \in\{1.75,1.50,1.25,1.05\}$, are considered. For each value of $\beta, \lambda=0.25$ and $A_{0}$ and $A_{1}$ are such that $A_{0} \int_{\mathbf{R}}|x|^{1-\beta} e^{-\lambda|x|} d x=1$ and $A_{1} \int_{\mathbf{R}}|x|^{1-\beta / 3} e^{-\lambda|x|} d x=0.2$.

Next, four specifications in which $X_{t}$ contains a Brownian motion are considered. For all of the four cases JA index of $X_{t}$ is equal to 2. The four cases are considered in order to examine the robustness to jumps and stochastic volatility. In the first case, $X_{t}$ is a standard Brownian motion $\left(a=0, \sigma_{t}=1, \forall t, L_{t}=W_{t}\right)$. In the second case the volatility is stochastic and it is specified as in Hounyo and Varneskov (2017): $L_{t}$ is equal to a Brownian motion $W_{t}$ and $\sigma_{t}$ is assumed to follow a two-factor model:

$$
\sigma_{t}=\psi\left(b_{0}+b_{1} \tau_{1, t}+b_{2} \tau_{2, t}\right)
$$

where

$$
\psi(x)= \begin{cases}\exp (x), & x \leq x_{0} \\ \frac{\exp \left(x_{0}\right)}{\sqrt{x_{0}}} \sqrt{x_{0}-x_{0}^{2}+x^{2}}, & x>x_{0}\end{cases}
$$


with $x_{0}=\log (1.5)$,

$$
d \tau_{1, t}=a_{1} \tau_{1, t} d t+d B_{1, t}, \quad d \tau_{2, t}=a_{1} \tau_{2, t} d t+\left(1+\phi \tau_{2, t}\right) d B_{2, t},
$$

and both $B_{1, t}$ and $B_{2, t}$ are standard Brownian motions independent from each other. The drift and the parameters of stochastic volatility are equal to the values estimated by Huang and Tauchen (2005): $a=0.03, b_{0}=-1.2, b_{1}=0.04, b_{2}=1.5, a_{1}=-0.00137, a_{2}=-1.386$, $\phi=0.25, \operatorname{Corr}\left(B_{i, t}, W_{t}\right)=-0.3, i=1,2$. For each simulated path the most persistent volatility factor, $\tau_{1, t}$, is initialized by drawing from its unconditional distribution, $\tau_{1,0} \sim$ $\mathcal{N}\left(0,1 /\left(2 a_{1}\right)\right)$, and the strongly mean-reverting factor, $\tau_{2, t}$, is initialized at zero. In the last two cases, in addition to stochastic volatility, $X_{t}$ contains jumps: $L_{t}=W_{t}+J_{t}$. $J_{t}$ is specified as either a compound Poisson process with unit intensity and jump sizes equal to five local standard deviations or a pure-jump tempered stable process with unit JA index such that on average the total contribution of jumps to the quadratic variation of $X$ constitutes $25 \%$.

For each data-generating process the four sampling frequencies are considered: $n=$ $39,78,144,288$, which roughly correspond to five-minute and 10-minute returns observed over a single 6.5 and 24 hours trading day respectively. $n=144$ and $n=288$ are equal to the number of observations used in the empirical application in Section 5.

The JA index is estimated using the proposed two-step multipower-based estimator. For comparison, the estimation methods of Andersen et al. (2015) and Todorov (2015) are considered. The first method is the extended two-scale estimator of Todorov and Tauchen (2010), defined as

$$
\widehat{\beta}^{T T}(p)=\frac{p \log (2)}{\log (V(X ; p, 2))-\log (V(X ; p, 1))} \times \mathbf{1}_{\{V(X ; p, 2) \neq V(X ; p, 1)\}},
$$

where

$$
V(X ; p, 1)=\sum_{i=1}^{n}\left|\Delta_{i} X\right|^{p}, \quad V(X ; p, 2)=\sum_{i=2}^{n}\left|\Delta_{i} X+\Delta_{i-1} X\right|^{p},
$$

and $p>0$ is a tuning parameter. As in the empirical part of the work of Andersen et al. (2015), tree choices of $p$ are considered: $p \in\{0.5,0.7,0.9\}$. The second method (Todorov, 2015) is based on matching the real part of the empirical characteristic function of the increments with its limiting value. The JA estimator is defined as

$$
\hat{\beta}(p, \mathbf{u})=\operatorname{argmin}_{\beta \in(1,2]} \hat{\mathbf{m}}\left(p, \hat{\mathbf{u}}, \hat{\beta}^{f s}, \mathbf{u}, \beta\right)^{\prime} \mathbf{W}^{-1}\left(p, \hat{\mathbf{u}}, \hat{\beta}^{f s}\right) \hat{\mathbf{m}}\left(p, \hat{\mathbf{u}}, \hat{\beta}^{f s}, \mathbf{u}, \beta\right),
$$


where $\hat{\mathbf{m}}\left(p, \hat{\mathbf{u}}, \hat{\beta}^{f s}, \mathbf{u}, \beta\right)$ is $K \times 1$ vector of "moment restrictions" and $\mathbf{W}(p, \hat{\mathbf{u}}, \hat{\beta}$ "ss $)$ is a $K \times K$ weighting matrix. $\hat{\mathbf{m}}\left(p, \hat{\mathbf{u}}, \hat{\beta}^{f s}, \mathbf{u}, \beta\right)$ depends on a tuning parameter $p$, a preliminary consistent JA estimator $\hat{\beta}^{f s}$ and on an (adaptively chosen) range of values, specified by $\hat{\mathbf{u}}$ and $\mathbf{u}$, over which the empirical characteristic function is matched with its limit. This estimator is implemented using the same tuning parameters as in the original paper Todorov (2015). In the tables it is denoted as $\widehat{\beta}^{T}$.

Table 2 presents the simulation results when $X_{t}$ is a mixture of tempered stable processes. It reports bias, standard deviation and root mean squared error (RMSE) for different estimators and activity indexes. The new multipower-based JA estimator performs well for all considered sampling frequencies and jump activity levels. At the lowest frequency $(n=39)$ it outperforms the benchmarks in both bias and variance for all considered JA indexes. At the highest considered frequency $(n=288)$, the Todorov (2015) estimator is comparable with the new one. The performance of the two-scale alternative is considerably worse than the new estimator, especially for lower sampling sizes and higher JA indexes. For example, when $\beta=1.75$ and $n=39$, the RMSE of the new $\widehat{\beta}$ is 0.293 , while it is equal to 4.340 for the two-scale $\widehat{\beta}^{T T}(0.90)$ (which is roughly 14 times larger); when $\beta=1.75$ and $n=288$, the RMSE of $\widehat{\beta}$ is 0.129 , while the RMSE of $\widehat{\beta}^{T T}(0.90)$ is 0.196 (which is roughly $50 \%$ larger).

The simulation results for the cases in which $X_{t}$ contains a Brownian component are reported in Table 3 . When $X_{t}$ is generated by a Brownian motion, the performance of $\widehat{\beta}$ is almost as good as the performance of the characteristic-function based estimator: $\widehat{\beta}$ has slightly smaller bias but slightly larger variance. However, the new estimator is more robust than $\widehat{\beta}^{T}$ to the presence of stochastic volatility and jumps. For example, when $n=144$ and volatility is constant, the standard deviation of $\widehat{\beta}^{T}$ is equal to 0.100 , while it is equal to 0.123 for $\widehat{\beta}$; under the stochastic volatility the standard deviation of $\widehat{\beta}^{T}$ increases up to 0.146 (roughly by 50\%), while the standard deviation of the new estimator increases up to 0.131 (roughly by $6.5 \%$ ). Moreover, stochastic volatility exacerbates the finite-sample bias of $\widehat{\beta}^{T}$. For example, when $n=39$ the bias of $\widehat{\beta}^{T}$ is -0.168 , while it is just -0.015 for the new estimator. Both $\widehat{\beta}$ and $\widehat{\beta}^{T}$ are significantly outperform the two-scale estimator, which appears to be very volatile and positively biased. For example, when $n=78$ and 
Table 2: Estimates of $\beta$ for a mixture of tempered stable processes.

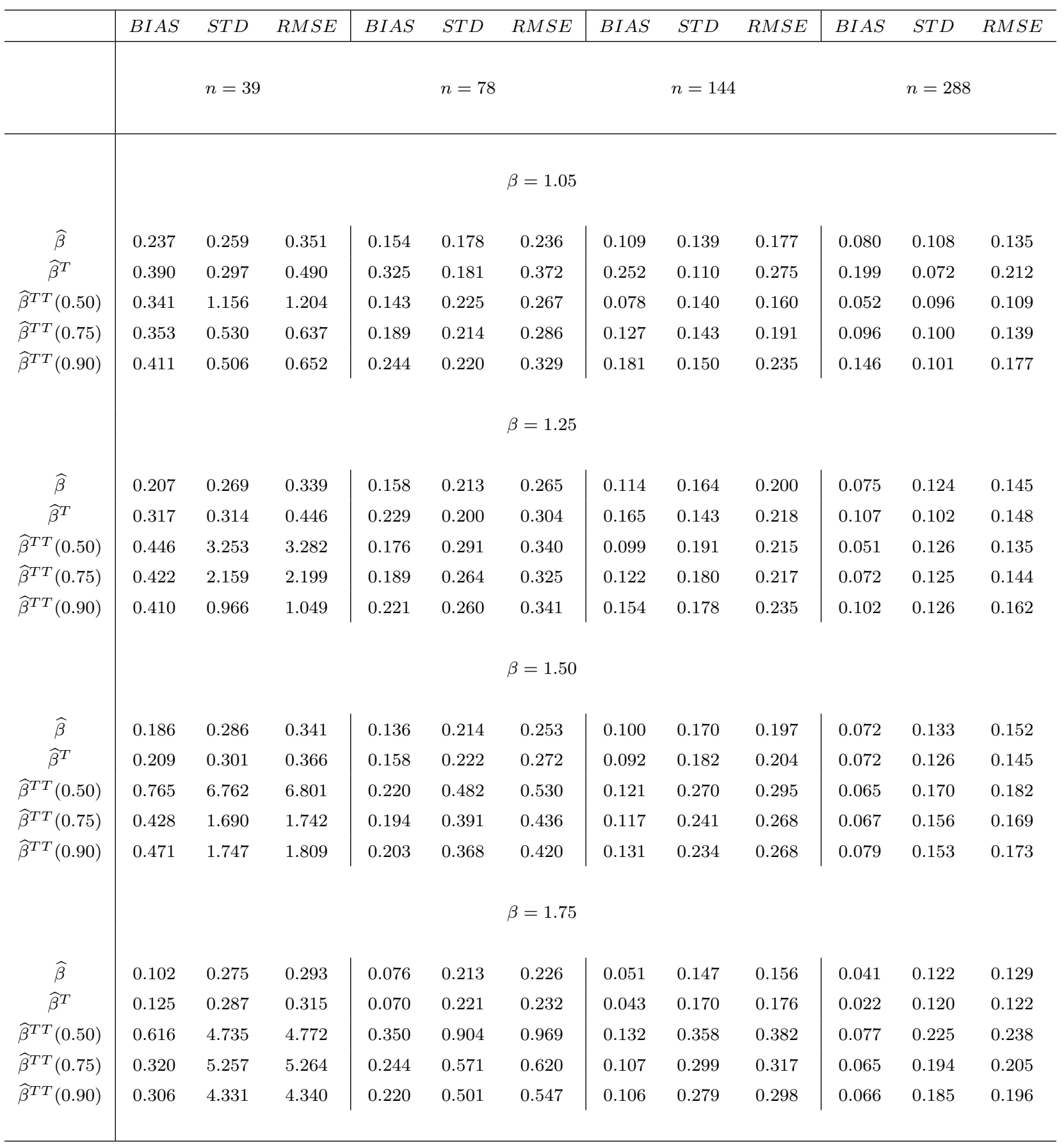

Note. The table reports the sample biases, standard deviations and root mean squared errors for different JA index estimators.

The data-generating process is specified as a mixture of tempered stable processes. 
Table 3: The estimates of $\beta$ in models driven by Brownian motion.

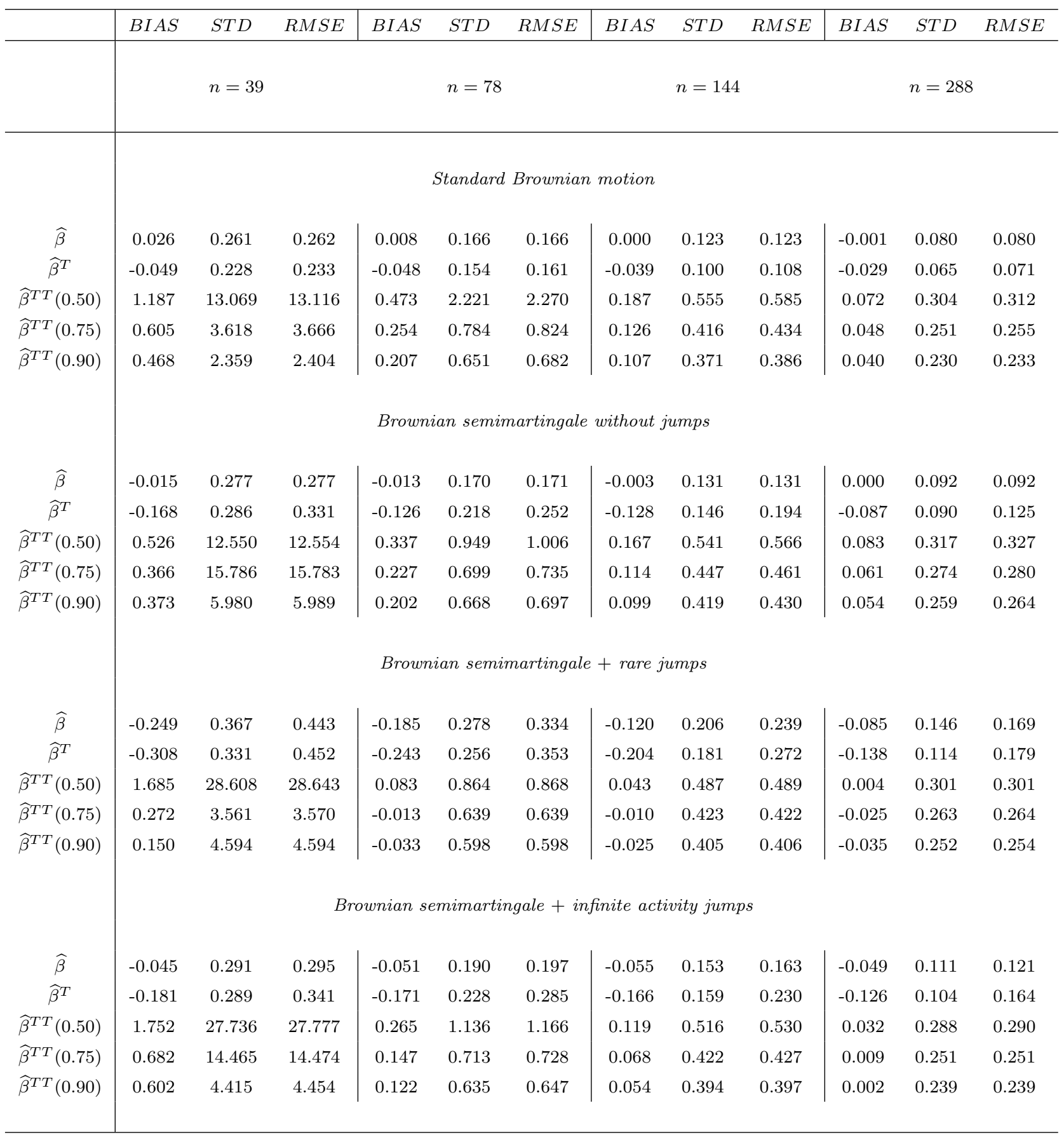

Note. The table reports the sample biases, standard deviations and root mean squared errors for different JA index estimators.

The data-generating process contains a Brownian component, hence JA $=2$ for all panels. 
the volatility is stochastic, the bias and standard deviation of the two-scale $\widehat{\beta}^{T T}(0.90)$ are respectively 0.202 and 0.668 , while they are -0.013 and 0.170 for the new estimator. The presence of (either finite or infinite activity) jumps increases the variance of all estimators and introduces a negative finite sample bias, which can be particularly strong for smaller $n$. The new estimator is again more robust with respect to the benchmarks. For example, when $n=39$ and jumps are of infinite activity, the bias of $\widehat{\beta}$ is -0.045 , while it is -0.181 for the estimator of Todorov (2015).

Altogether the results of Monte Carlo experiments suggest that the new multipowerbased JA estimator is sufficiently precise for different data-generating schemes. It is more robust across different data-generating processes than the benchmark estimators and typically outperforms them in short samples. Together with the simplicity of the implementation this allows estimating jump activity using even moderate frequency data (as, e.g. five- or 10-minute data), which is particularly useful for financial applications, in which sampling asset prices at higher frequencies may result in a distortion due to the market microstructure noise.

Now, to examine the size and power of the test introduced in Section 3.4, it is applied to the simulated data. In order to enlarge the power, the test statistics are computed weekly (the data are pooled over 5 intervals corresponding to 24-hours trading days) with $n=288$ observations for each day, as is done in the empirical application in Section 5 . Figure 2 displays the number of rejections of $\mathcal{H}_{0}: \beta \leq \beta^{\diamond}$ for a range of $\beta^{\diamond}$ 's and for the two data-generating processes. The right panel reports the size (the points corresponding to $\beta^{\diamond}=2$ ) and the power (the points corresponding to $\beta^{\diamond}<2$ ) of the test against the Brownian semimartingale without jumps specified above. The power of the test is nearly perfect when $\beta^{\diamond}<1.7$ and reasonably large for $\beta^{\diamond}<1.8$. Rejecting $\mathcal{H}_{0}$ with larger $\beta^{\diamond}$ is more challenging: for example, with $\beta^{\diamond}=1.9$ the rejection rates are roughly $16 \%$ and $59 \%$ with $99 \%$ and $90 \%$ confidence levels respectively. The size of the test coincides with the theoretical level. The left panel of Figure 2 displays the rejections of $\mathcal{H}_{0}$ for the mixture of tempered stable processes with JA index 1.75. The rejection rate exceeds $90 \%$ for $\beta^{\diamond}<1.5$. For $\beta^{\diamond}=1.70$, the $\mathcal{H}_{0}$ rejection rates are roughly $5 \%$ and $30 \%$ with $99 \%$ and $90 \%$ confidence levels respectively. The number of rejections of $\mathcal{H}_{0}$ when $\beta^{\diamond}$ is larger than the true JA index 

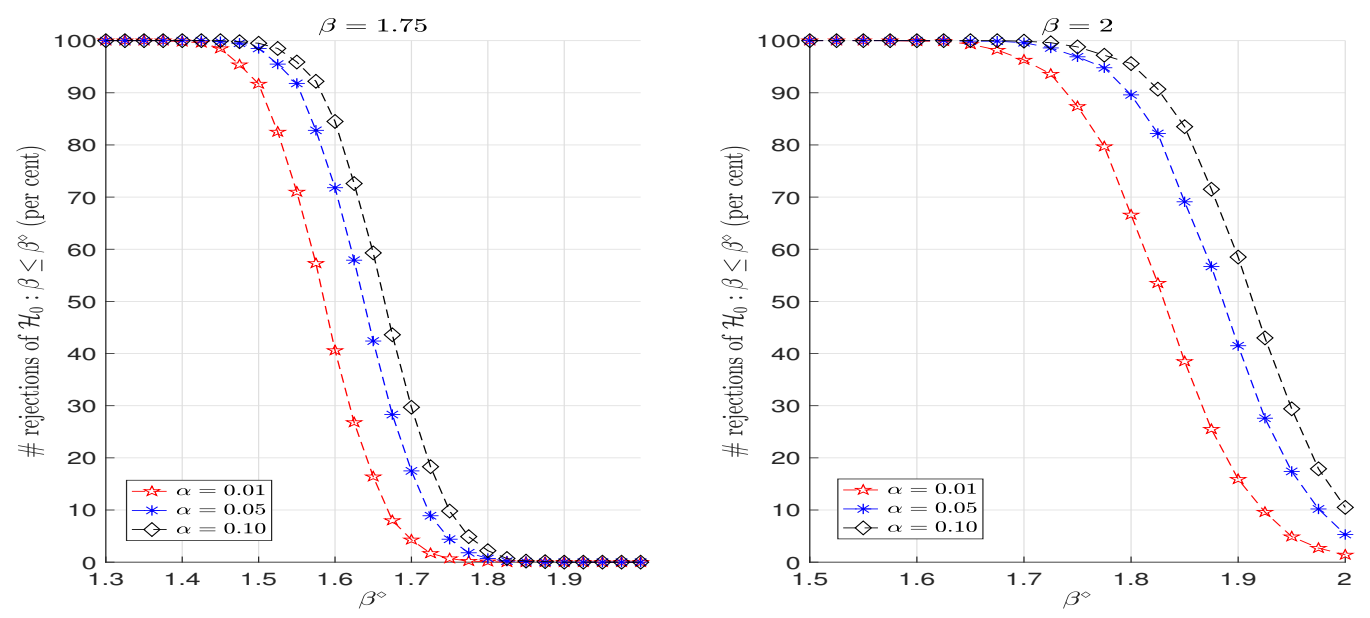

Figure 2: The number of rejections of $\mathcal{H}_{0}: \beta \leq \beta^{\diamond}$ (per cent) with the size $\alpha$ for different values of $\beta^{\diamond}$ based on weekly estimates of JA index from the data generated by the mixture of tempered stable processes with JA index 1.75 (left panel) or by the Brownian semimartingale without jumps (right panel).

does not exceed the theoretical size of the test and quickly approaches zero as $\beta^{\diamond}$ increases.

\section{Empirical application}

Are cryptocurrencies different from other currencies and financial assets? A recent study by Scaillet et al. (2020) shows that common jump detection techniques applied to intraday bitcoin data reveal a significantly larger number of jumps and the jump dynamics is significantly different from what previous research observes for large-cap assets and indices. These findings ought to be expected if bitcoin follows a pure-jump process. Hence, in the present paper, in order to examine the pure-jump hypothesis, the jump activity of bitcoin is investigated. Bitcoin is the most prominent example of a cryptocurrency, that is a peer-topeer digital asset, which is decentralized and allows the execution of transactions directly between users. The technology behind bitcoin and its structure is described in details, for example, by Böhme et al. (2015).

Estimation of the JA index of bitcoin is based on high-frequency returns of bitcoin/US dollar exchange rate (BTC/USD). The data are available from the leading brokerage firm 
Table 4: Summary statistics for the data

\begin{tabular}{l|ccccccccc}
\hline & Mean & Std & Skew & Kurt & $q_{0.25}\left(\frac{R V_{10}}{R V_{5}}\right)$ & $q_{0.5}\left(\frac{R V_{10}}{R V_{5}}\right)$ & $q_{0.75}\left(\frac{R V_{10}}{R V_{5}}\right)$ & $R Z_{5}$ & $R Z_{10}$ \\
\hline BTC/USD & 0.304 & 3.479 & -0.334 & 11.188 & 0.887 & 1.013 & 1.295 & 6.702 & 3.896 \\
EUR/USD & 0.004 & 0.516 & 0.416 & 7.066 & 0.871 & 0.964 & 1.060 & 2.026 & 1.368 \\
\hline \hline
\end{tabular}

Note. This table reports summary statistics of BTC/USD and EUR/USD exchange rates: mean, standard deviation, skewness and kurtosis of daily logarithmic returns (per cent), quintiles of the ratio of daily realized variances computed at five- and 10 -minute scales and the average daily percentage of zero returns at five- and 10-minute frequency (respectively $R Z_{5}$ and $\left.R Z_{10}\right)$.

in Russia Finam (at www.finam.ru). The dataset spans the period from February 12, 2015 to September 19, 2017, for a total of 946 trading days. For each trading day (which is 24 hours), the data consists of five-minute records of BTC/USD, corresponding to 288 observations. For comparison, five-minute records of the EUR/USD exchange rate during the same sample period are considered.

Table 4 reports simple summary statistics of the data. The sample mean and standard deviation of daily bitcoin returns are extraordinary large, as it exhibits an abnormal bubbling growth over the considered sample period. High kurtosis indicates the heavy tails. The sample quantiles of the ratio of the daily realized variances (RV) computed at the fiveand 10-minute scales suggest that the data are unlikely to be heavily polluted by the microstructure noise (since RV is invariant to the sampling frequency in the absence of noise). A significant fraction of intraday returns are equal to zero, indicating the price staleness phenomenon documented for stock prices (Bandi et al., 2019). On average, 6.70\% of fiveminute and $3.90 \%$ of 10-minute returns are zero as for the most liquid stocks (for example, the daily percentage of zero returns in stock prices belonging to the S\&P 500 index for a period from January 1998 to June 2015 is 14.78\% when sampling at five minute). Figure 3 illustrates the time-series of daily records of BTC/USD (left panel) and an example of the intraday dynamics (right panel). On that day BTC/USD exhibits several very large movements, which can be characterized as jumps. At the same time, many five-minute changes are very small (almost zero). Such a difference between large and small movements is typical for a pure-jump process. Indeed, the estimated jump activity index at that day is equal to 1.636 . 

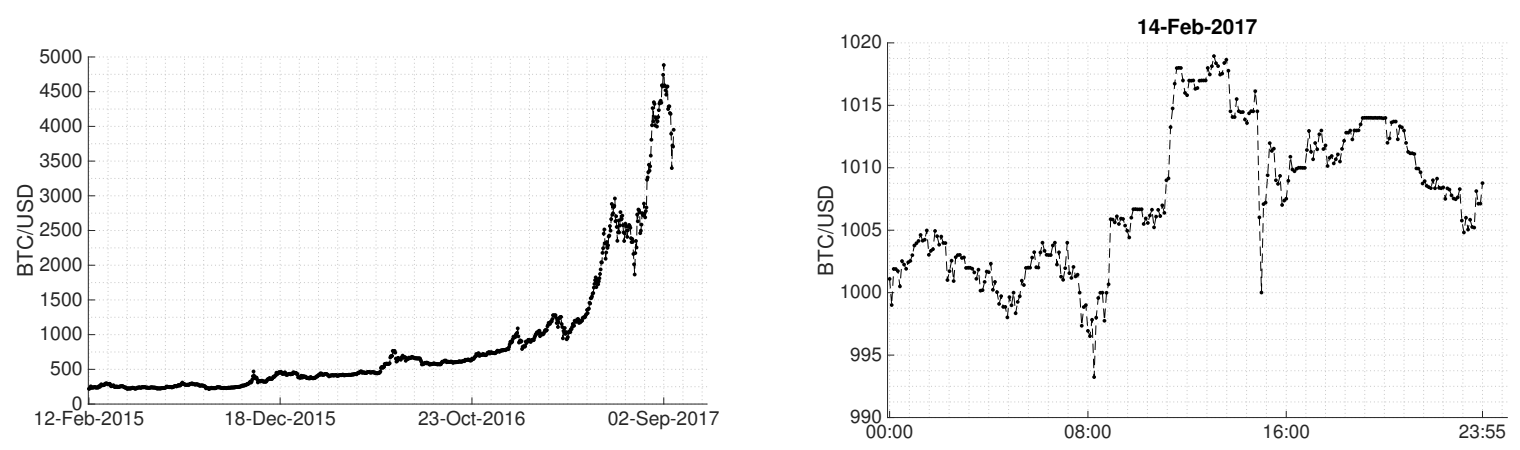

Figure 3: The left panel illustrates daily BTC/USD exchange rate dynamics from February 12, 2015 to September 19, 2017. The right panel plots five-minute records of BTC/USD for February 14, 2017. The estimated JA index for that day is equal to 1.636.

The JA index is estimated using the two-step estimator of Section 3.3. In order to mitigate the potential effect of market microstructure noise five- and 10-minute returns are used. Days with more than $30 \%$ of zero returns are excluded from consideration. For the remaining days the multipower variations are computed leaving out zero returns, as explained in the supplementarity Appendix. At both five- and 10-minute frequencies the first step estimator uses $r_{+}=0.75$. On average this leads to the second-step value of $r_{+}$ being 0.874 and 0.890 for respectively five- and 10-minutes BTC/USD returns and 0.988 and 0.980 for respectively five- and 10-minutes EUR/USD returns.

Figure 4 displays kernel smooth densities of daily JA index estimates of BTC/USD and EUR/USD exchange rates. The jump activity of the two rates are remarkably different. The JA index of EUR/USD is close to 2, indicating the presence of a continuous martingale component. This confirms previous findings regarding the high-frequency dynamics of exchange rates (Todorov and Tauchen, 2010; Cont and Mancini, 2011). In contrast, the estimates of the JA index of bitcoin are centered at a value of 1.8, indicating pure-jump dynamics.

Summary statistics of the point JA estimates are reported in Table 5. Bitcoin JA index estimates based on five-minute frequency range between 1.275 and 2.131 with average value of 1.767 , which would be as expected for a pure-jump process with a high activity level. These indications appear to be consistent over sampling frequencies: if 10-minute returns 


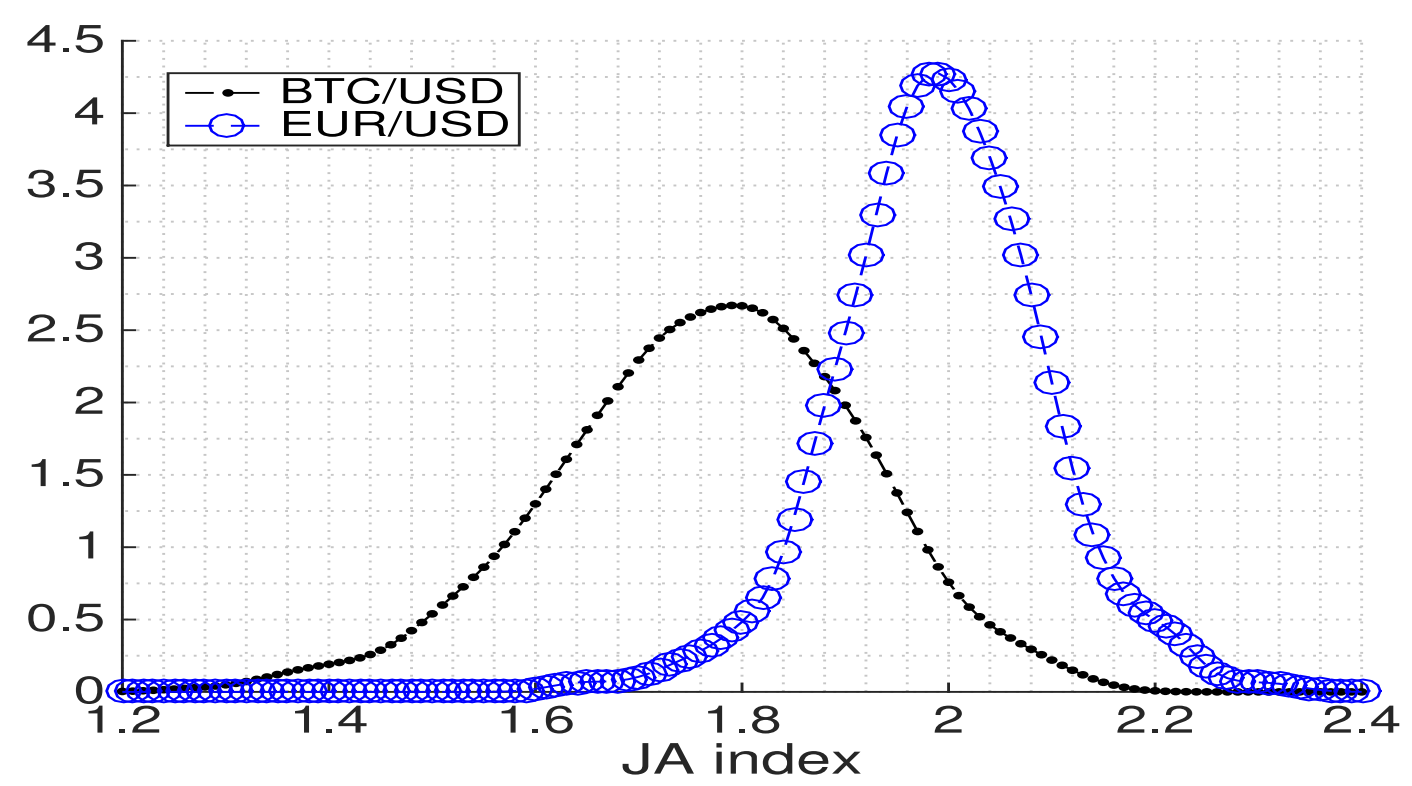

Figure 4: Kernel smooth densities of daily JA index estimates of BTC/USD and EUR/USD exchange rates. The JA indexes are estimated by the two-step estimator of Section 3.3, using five-minute returns.

are used for JA index estimation, the average value of the estimates is equal to 1.808 , while maximum and minimum estimates are equal respectively to 1.270 and 2.396.

Next, the hypothesis $\mathcal{H}_{0}: \beta \leq \beta^{\diamond}$ is tested (against the alternative $\mathcal{H}_{1}: \beta>\beta^{\diamond}$ ) with $\beta^{\diamond} \in\{1.5,1.8\}$ using the criterion developed in Section 3.4. The test statistics is computed weekly (the five-minute data are pooled over five consecutive trading days). Table 6 reports the number of rejections at different significance levels. For bitcoin, the number of rejections of $\mathcal{H}_{0}: \beta \leq 1.5$ varies from $45 \%$ to $63 \%$ depending on the significance level, while the number of rejections of $\mathcal{H}_{0}: \beta \leq 1.8$ roughly coincide with the theoretical type-one error. For comparison, the right panel of Table 6 shows that for EUR/USD the null $\mathcal{H}_{0}: \beta \leq 1.5$ is rejected for $100 \%$ of the conducted tests. The number of rejections of $\mathcal{H}_{0}: \beta \leq 1.8$ varies from $71 \%$ to $94 \%$, as one would expect under the presence of Brownian motion (see Figure 2). Hence, the statistical testing supports the evidence provided by the point JA estimates.

For comparison and as a robustness check the jump activity of the two exchange rates are estimated using the two-scale estimator of Andersen et al. (2015) defined by the equation 
Table 5: Summary statistics of point JA index estimates.

\begin{tabular}{l|rrrrr}
\hline & Mean & Median & Std & min & max \\
\hline & \multicolumn{5}{|c}{ five-minute frequency } \\
BTC/USD & 1.767 & 1.775 & 0.146 & 1.275 & 2.131 \\
EUR/USD & 1.993 & 1.994 & 0.099 & 1.626 & 2.321 \\
& \multicolumn{5}{c}{$\mathbf{1 0 - m i n u t e}$ frequency } \\
BTC/USD & 1.808 & 1.807 & 0.153 & 1.270 & 2.396 \\
EUR/USD & 2.002 & 2.000 & 0.137 & 1.611 & 2.458 \\
\hline \hline
\end{tabular}

Note. The table reports means, medians, sample standard deviations, minimum and maximum values of JA index estimates $\widehat{\beta}$ computed at 5 -and 10-minute scales.

Table 6: Rejections of $\mathcal{H}_{0}: \beta \leq \beta^{\diamond}$.

\begin{tabular}{l|ccc|ccc}
\hline size $\rightarrow$ & $\alpha=0.10$ & $\alpha=0.05$ & $\alpha=0.01$ & $\alpha=0.10$ & $\alpha=0.05$ & $\alpha=0.01$ \\
\hline & \multicolumn{3}{|c|}{ BTC/USD } & \multicolumn{4}{c}{ EUR/USD } \\
$\beta^{\diamond}=1.5$ & 63.830 & 57.979 & 45.745 & 100.000 & 100.000 & 100.000 \\
$\beta^{\diamond}=1.8$ & 8.511 & 5.319 & 2.660 & 94.416 & 88.325 & 71.066 \\
\hline \hline
\end{tabular}

Note. The table reports the number of rejections of $\mathcal{H}_{0}: \beta \leq \beta^{\diamond}$ for $\beta^{\diamond} \in\{1.5,1.8\}$ and for different significance levels $\alpha$. 
(40). Figure 5 compares the kernel-smooth densities of two-scale and multipower-based activity estimates. First, it illustrates that for both exchange rates the variation of the two-scale estimates is much larger with respect to the multipower-based ones (for instance, standard deviation of the two-scale BTC/USD JA index estimates is 0.521, while it is equal to 0.146 for the multipower-based estimates). Second, the kernel smooth densities of the two estimates peak around the same value, but two-scale estimates exhibit a pronounced fat right tail. As a result, the sample average and median of the two-scale estimates are very different. For example, for BTC/USD, the average value of the estimates is equal to 1.992, but the median value is 1.876 . The presence of this right tail is consistent with the fact that two-scales estimator may exhibit a large positive bias in finite samples, as shown in the Monte Carlo section. Taking this into account, it is possible to conclude that the application of two-scale estimator to the real data qualitatively confirms the findings based on the new method: the activity of EUR/USD is larger then the activity of BTC/USD; the dynamics EUR/USD contains a Brownian component, while BTC/USD is better described by a pure-jump process with high jump activity. These findings, however, could hardly be discovered by only considering the two-scale estimator.

Overall, the analysis suggests that bitcoin is a pure-jump process without a continuous component and with a relatively high JA index. This result has several important practical implications. First, the risks of bitcoin could be far beyond what is measured by realized variance. Realized measures, such as realized covariances, do not admit usual interpretation when being applied to cryptocurrencies. Therefore, one ought to be particularly careful regarding measuring the risks when adding bitcoin to a currency portfolio. Second, widespread jump-diffusion models might be not suitable for parametric modeling of cryptocurrencies. Instead, pure-jump models, for example, based on time-changed Brownian motion, are more appropriate for parametric modeling, for example, for pricing cryptocurrency derivatives.

\section{Concluding remarks}

This paper proposes a new method for estimating the jump activity index of a discretely observed process based on comparing realized multipower variations with different powers. 

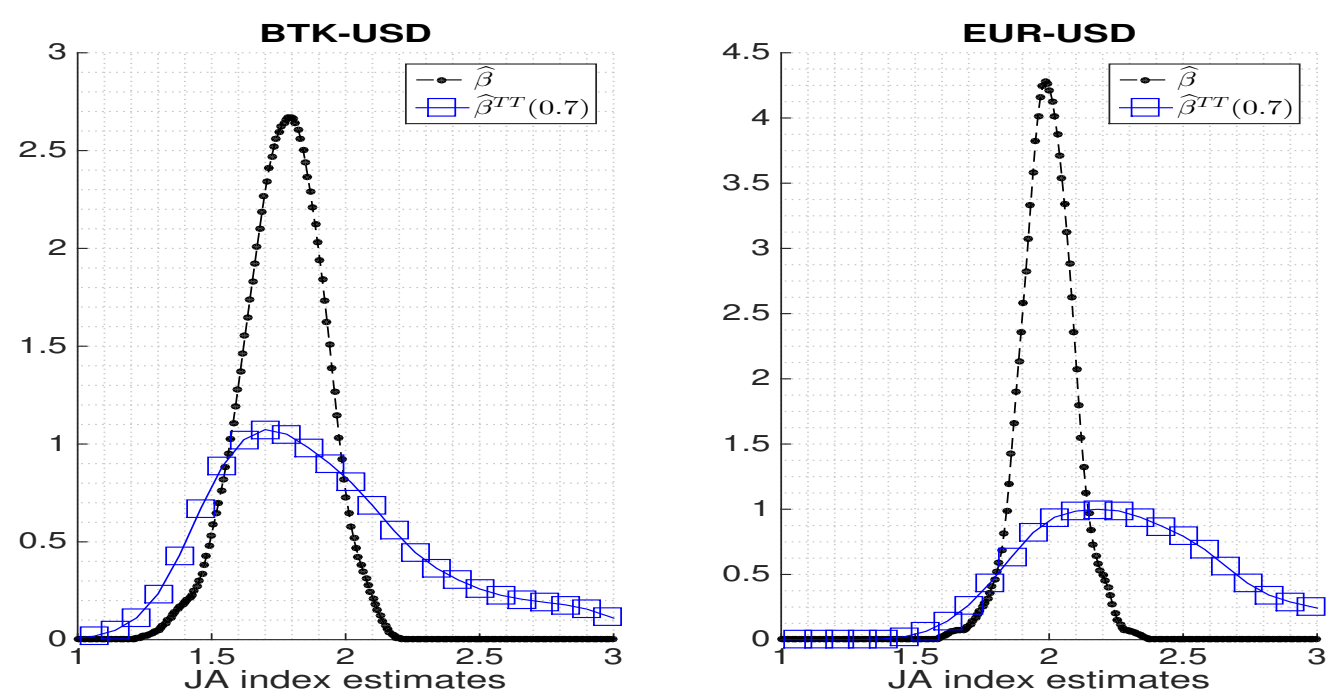

Figure 5: Kernel smooth densities of daily estimates of JA index of BTC/USD and EUR/USD computed at five-minute frequency using the new multipower-based estimator, $\widehat{\beta}$, and the two-scale estimator, $\widehat{\beta}^{T T}(p)$, defined by the equation (40), $p=0.7$.

As shown on simulated data, the new jump activity estimator outperforms the benchmarks. Due to the good finite sample precision and the simplicity of its implementation, the new estimation method is not only relevant for complementing existing empirical research, but could also be used as a leading instrument for verifying the presence of a Brownian component in an observed process. For example, the empirical part of the paper provides clear-cut evidence that bitcoin is a pure-jump process, which contrasts bitcoin from conventional currencies that include a Brownian motion. Verifying this result without using the new estimator is not straightforward.

While in the recent econometric literature realized multipower variations are primarily used to eliminate jumps, this paper shows that multipowers can be used for inference for pure-jump processes instead. Analogously, the idea of comparing multipower variations with different powers could serve as an alternative to comparing power variations computed over different scales in other applications. 


\section{References}

Ait-Sahalia, Y., Jacod, J., 2009. Estimating the degree of activity of jumps in high frequency financial data. Annals of Statistics 37, 2202-2244.

Aït-Sahalia, Y., Jacod, J., 2010. Is Brownian motion necessary to model high frequency data? Annals of Statistics 38, 3093-3128.

Andersen, T., Benzoni, L., 2011. Stochastic volatility, in: Complex Systems in Finance and Econometrics. Springer, pp. 694-726.

Andersen, T.G., Bondarenko, O., Todorov, V., Tauchen, G., 2015. The fine structure of equity-index option dynamics. Journal of Econometrics 187, 532-546.

Bandi, F., Kolokolov, A., Pirino, D., Renó, R., 2019. Zeros. Management Science, forthcoming.

Barndorff-Nielsen, O., 1998. Processes of normal inverse Gaussian type. Finance and Stochastics 2, 41-68.

Barndorff-Nielsen, O., Graversen, E., Jacod, J., Shephard, N., 2006a. Limit theorems for bipower variation in financial econometrics. Econometric Theory 22, 677-719.

Barndorff-Nielsen, O., Shephard, N., 2003. Realized power variation and stochastic volatility models. Bernoulli 9, 243-265.

Barndorff-Nielsen, O., Shephard, N., 2004. Power and bipower variation with stochastic volatility and jumps. Journal of Financial Econometrics 2, 1-48.

Barndorff-Nielsen, O., Shephard, N., 2006. Econometrics of testing for jumps in financial economics using bipower variation. Journal of Financial Econometrics 4, 1-30.

Barndorff-Nielsen, O., Shephard, N., Winkel, M., 2006b. Limit theorems for multipower variation in the presence of jumps. Stochastic Processes and their Applications 116, $798-806$.

Böhme, R., Christin, N., Edelman, N., Moore, T., 2015. Bitcoin: Economics, technology, and governance. The Journal of Economic Perspectives 29, 213-238.

Carr, P., Geman, H., Madan, D., Yor, M., 2002. The fine structure of asset returns: An empirical investigation. Journal of Business 75, 305-332.

Carr, P., Geman, H., Madan, D., Yor, M., 2003. Stochastic volatility for Lévy processes. 
Mathematical Finance 58, 345-382.

Carr, P., Wu, L., 2003. The finite moment log stable process and option pricing. The Journal of Finance LVIII, 753-778.

Cont, R., Mancini, C., 2011. Nonparametric tests for pathwise properties of semimartingales. Bernoulli 17, 781-813.

Corsi, F., Pirino, D., Renó, R., 2010. Threshold bipower variation and the impact of jumps on volatility forecasting. Journal of Econometrics 159, 276-288.

Hounyo, U., Varneskov, R.T., 2017. A local stable bootstrap for power variations of purejump semimartingales and activity index estimation. Journal of Econometrics 198, 10-28.

Huang, J., Wu, L., 2004. Specification analysis of option pricing models based on timechanged Lévy processes. Journal of Finance 59, 1405-1439.

Huang, S., Hung, M.W., 2005. Pricing foreign equity options under Lévy processes. Journal of Futures Markets 25, 917-944.

Huang, X., Tauchen, G., 2005. The relative contribution of jumps to total price variance. Journal of Financial Econometrics 3, 456-499.

Jacod, J., 2008. Asymptotic properties of realized power variations and related functionals of semimartingales. Stochastic Processes and their Applications 118, 517-559.

Jacod, J., Protter, P., 2012. Discretization of Processes. Springer-Verlag.

Jing, B.Y., Kong, X.B., Liu, Z., 2011. Estimating the jump activity index under noisy observations using high-frequency data. Journal of the American Statistical Association $106,558-568$.

Jing, B.Y., Kong, X.B., Liu, Z., 2012a. Modeling high-frequency financial data by pure jump processes. The Annals of Statistics 40, 759-784.

Jing, B.Y., Kong, X.B., Liu, Z., Mykland, P., 2012b. On the jump activity index for semimartingales. Journal of Econometrics 166, 213-223.

Madan, D., Carr, P., Chang, E., 1998. The variance Gamma process and option pricing. European Finance Review 2, 79-105.

Mancini, C., 2009. Non-parametric threshold estimation for models with stochastic diffusion coefficients and jumps. Scandinavian Journal of Statistics 36, 270-296.

Rydberg, T.H., 1997. The normal inverse Gaussian Lévy process: Simulation and approx- 
imation. Comm. Statist. Stochastic Models 13, 887-910.

Scaillet, O., Treccani, A., Trevisan, C., 2020. High-frequency jump analysis of the bitcoin market. Journal of Financial Econometrics 18, 209-232.

Shanbhag, D., Sreehari, M.Z., 1977. On certain self-decomposable distributions. Wahrscheinlichkeitstheorie verw Gebiete 38, 217-222.

Todorov, V., 2015. Jump activity estimation for pure-jump semimartingales via selfnormalized statistics. Annals of Statistics 43, 1831-1864.

Todorov, V., Tauchen, G., 2010. Activity signature functions for high-frequency data analysis. Journal of Econometrics 154, 125-138.

Todorov, V., Tauchen, G., 2011a. Limit theorems for power variations of pure-jump processes with application to activity estimation. Annals of Applied Probability 21, 546-588.

Todorov, V., Tauchen, G., 2011b. Volatility jumps. Journal of Business and Economic Statistics 29, 356-371.

Vetter, M., 2010. Limit theorems for bipower variation of semimartingales. Stochastic Processes and their Applications 120, 22-38.

Woerner, J., 2003. Variational sums and power variation: A unifying approach to model selection and estimation in semimartingale models. Statistics and Decisions 21, 47-68.

Woerner, J., 2007. Inference in Lévy-type stochastic volatility models. Advances in Applied Probability 39, 531-549.

Wu, L., 2006. Dampened power law: Reconciling the tail behavior of financial security returns. Journal of Business 79, 1445-1473. 